\title{
AVALIAÇÃO HIGROSCÓPICA DA FIBRA RESIDUAL DO ABACAXI
}

\section{HYGROSCOPIC EVALUATION OF PINEAPPLE RESIDUAL FIBER}

\author{
Tonye Gil Matos Waughon ${ }^{1}$; Rosinelson da Silva Pena ${ }^{2}$ \\ ${ }^{1}$ Escola de Trabalho e Produção de Itaituba - Itaituba - PA - Brasil tonyegil@hotmail.com \\ ${ }^{2}$ DEQAL/CT/UFPA - Campus Universitário do Guamá - Belém - PA - Brasil rspena@ufpa.br
}

\begin{abstract}
Resumo
Avaliou-se o comportamento higroscópico da fibra residual do abacaxi, resíduo da industrialização do suco concentrado; após secagem em secador de leito fixo. Foram construídas isotermas de adsorção e dessorção de umidade a $25^{\circ} \mathrm{C}$ e $40^{\circ} \mathrm{C}$. Determinou-se o valor da monocamada BET $\left(m_{o}\right)$ e testou-se a aplicabilidade de doze modelos matemáticos na predição dos dados de sorção. O produto apresentou isotermas de sorção do Tipo III. Os valores médios da monocamada $\left(m_{o}\right)$ para adsorção e dessorção foram de 11,6 g $\mathrm{H}_{2} \mathrm{O} / 100 \mathrm{~g}$ b.s. e 12,7 $\mathrm{g} \mathrm{H} \mathrm{H}_{2} \mathrm{O} / 100 \mathrm{~g}$ b.s., respectivamente. Entre os modelos matemáticos testados, podem ser utilizados na predição das isotermas de sorção de umidade da fibra residual do abacaxi, nas condições estudadas e com margens de erro aceitáveis, os modelos bi-paramétricos de Oswin, Halsey e Mizrahi, e os modelos tri-paramétricos de GAB, BET modificada, Hailwood-Horrobin, Anderson e Anderson-Hall.
\end{abstract}

Palavras-chave: isotermas; sorção; resíduo; abacaxi.

\section{Introdução}

A umidade de um alimento é expressa pelo valor obtido na determinação da água total contida no mesmo. Entretanto, esse valor não fornece indicações de como a água está distribuída, bem como não permite saber se toda ela está ligada da mesma maneira. O conhecimento da distribuição da água no alimento é mais importante que simplesmente o conhecimento da umidade.

O parâmetro que avalia a forma como a água está distribuída em um alimento é a atividade de água $\left(\mathrm{a}_{\mathrm{w}}\right)$, a qual é uma medida da quantidade de moléculas de água livres ou ativas; geralmente obtida em relação à pressão de vapor da água pura. Esta medida é de fundamental importância, pois permite predizer alterações químicas, enzimáticas e microbianas, além de viabilizar a proposição de sistemas adequados de embalagem, para determinado produto (Labuza, 1968).

As isotermas de sorção de umidade descrevem a relação entre umidade e $\mathrm{a}_{\mathrm{w}}$ de um produto, à temperatura constante. São importantes na concentração e desidratação de alimentos. Através das isotermas pode-se determinar a monocamada, a qual além de estar relacionada com o 
início de uma série de reações químicas de deterioração dos alimentos, representa o ponto de maior gasto de energia em um processo de secagem (Uboldi eiroa, 1981).

Muitas vezes, após a obtenção experimental da isoterma faz-se necessário a utilização de modelos matemáticos que facilitam a predição de um maior número de pontos, partindo de poucos pontos experimentais, devido à limitação de medida dos higrômetros, em baixos níveis de atividade de água $\left(\mathrm{a}_{\mathrm{w}}\right)$, ou ao rápido desenvolvimento de fungos em altos níveis de $\mathrm{a}_{\mathrm{w}}$ (Lomauro et al., 1985a; Lomauro et al., 1985b).

Na elaboração do suco de abacaxi é descartado, além da casca e a coroa, parte da polpa, rica em fibras. De acordo com estudos realizados por Waughon (2006) este resíduo, após secagem, pode ser utilizado, como complemento, na elaboração de produtos a serem empregados em dietas ricas em fibras.

Este trabalho tem como objetivo avaliar o comportamento higroscópico da fibra residual do abacaxi (Ananas comosus L.), gerada na industrialização do suco concentrado; após secagem, em camada delgada, em secador de leito fixo.

\section{Material e métodos}

\subsection{Matéria prima}

O material estudado foi o resíduo fibroso obtido na primeira filtração, durante a industrialização do suco de abacaxi (Ananas comosus L.); fornecido pela indústria Sucos da Amazônia Agroindustrial Ltda - SUCASA, instalada no município de Belém (PA). O resíduo bruto foi submetido à secagem em secador de leito fixo (camada delgada), em condições otimizadas (Waughon, 2006). Após secagem o material foi submetido à moagem, em moinho de facas. O produto, na forma de uma farinha fina, foi então submetido aos ensaios de adsorção e dessorção.

\subsection{Métodos}

\subsubsection{Obtenção dos dados de adsorção}

Amostras do produto seco e moído foram pesadas $(1 \pm 0,001 \mathrm{~g})$, em recipientes próprios do aparelho de $\mathrm{a}_{\mathrm{w}}$. Foram submetidas à secagem complementar, em dessecador com sílica gel, sob vácuo, à temperatura ambiente $\left(\approx 25^{\circ} \mathrm{C}\right)$ e por um período de 24 horas. Após este período as amostras foram colocadas em dessecador contendo água na base, o qual foi mantido em ambiente com temperatura controlada $\left(25^{\circ} \mathrm{C}\right.$ e $\left.40^{\circ} \mathrm{C}\right)$. Em tempos crescentes amostras foram retiradas (pseudo-equilíbrio), em duplicata, e pesadas para a determinação da umidade, por diferença de massa, e da $\mathrm{a}_{\mathrm{w}}$, com auxílio de um higrômetro AQUAlab 3TE. 


\subsubsection{Obtenção dos dados de dessorção}

As amostras do produto, após o processo de adsorção, foram mantidas no dessecador contendo água na base, na temperatura de trabalho $\left(25^{\circ} \mathrm{C}\right.$ e $\left.40^{\circ} \mathrm{C}\right)$, por 24 horas, para que ocorresse a umidificação das mesmas. Após esse período foram transferidas para um dessecador contendo sílica gel na base, o qual foi mantido à temperatura controlada $\left(25^{\circ} \mathrm{C}\right.$ e $\left.40^{\circ} \mathrm{C}\right)$. Foram efetuadas retiradas das amostras, em duplicata, em tempos crescentes (pseudo-equilíbrio), para determinar umidade e $\mathrm{a}_{\mathrm{w}}$, da mesma maneira que na obtenção dos dados de adsorção.

O dessecador contendo as amostras contidas foi mantido sob vácuo, em estufa incubadora para $\mathrm{DBO}$, onde as variações de temperatura não ultrapassaram $\pm 1^{\circ} \mathrm{C}$. Durante os ensaios de adsorção e dessorção as amostras foram submetidas à inspeção visual, para identificar possíveis alterações que eventualmente ocorressem, tais com: “caking”, escurecimento e crescimento de fungos.

Isotermas de adsorção e dessorção foram construídas a partir dos dados de umidade e $\mathrm{a}_{\mathrm{w}}$, obtidos nos ensaios de adsorção e dessorção, a $25^{\circ} \mathrm{C}$ e $40^{\circ} \mathrm{C}$.

\subsubsection{Determinação da monocamada}

A monocamada $\left(\mathrm{m}_{\mathrm{o}}\right)$ foi determinada através da forma linearizada do modelo de BET (Brunauer et al., 1938) (Equação 1), com o auxílio do aplicativo STATISTICA 5.5 para Windows (Statistica, 2000).

$$
\frac{a_{w}}{\left(1-a_{w}\right) \cdot m}=\frac{1}{m_{o} \cdot C}+\frac{(C-1)}{m_{o} \cdot C} \cdot a_{w}
$$

onde: $\mathrm{m}$ é umidade (g/100 g b.s.), $\mathrm{a}_{\mathrm{w}}$ atividade de água, $\mathrm{m}_{\mathrm{o}}$ monocamada (g/100 g b.s.) e C constante relacionada com o calor de sorção.

\subsubsection{Predição das isotermas de sorção}

Foram testadas as aplicabilidades de doze modelos matemáticos, seis bi-paramétricos e seis tri-paramétricos, na predição dos dados de sorção, os quais são apresentados na Tabela 1. Utilizouse no ajuste regressão não linear, com auxílio do aplicativo STATISTICA 5.5 para Windows (Statistica, 2000).

O parâmetro primário utilizado para avaliar os ajustes dos modelos aos dados experimentais foi o coeficiente de determinação $\left(r^{2}\right)$. Para os modelos com os maiores valores de $r^{2}$ 
foi determinado o desvio médio relativo (P), através da Equação 2, para subsidiar a seleção do melhor modelo, com aplicabilidade na predição dos dados de sorção do produto estudado.

$$
\mathrm{P}=\frac{100}{\mathrm{n}} \sum_{\mathrm{i}=1}^{\mathrm{n}} \frac{\left|\mathrm{m}_{\exp }-\mathrm{m}_{\text {pre }}\right|}{\mathrm{m}_{\exp }}
$$

onde: $\mathrm{m}_{\exp }$ e $\mathrm{m}_{\text {pre }}$ são as umidades experimental e predita e $\mathrm{n}$ o número de observações.

Tabela 1 - Modelos utilizados na predição dos dados de sorção do resíduo

\begin{tabular}{|c|c|c|c|}
\hline & Nome da Equação & Modelos & Referencia \\
\hline \multirow{6}{*}{ 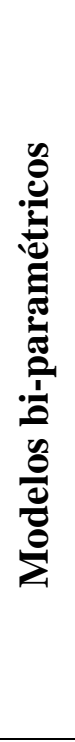 } & Kuhn & $\mathrm{m}=-\frac{\mathrm{a}}{\ln \mathrm{a}_{\mathrm{w}}}+\mathrm{b}$ & Chirife e Iglesias (1978) \\
\hline & Handerson & $\mathrm{m}=\left[\frac{-\ln \left(1-\mathrm{a}_{\mathrm{w}}\right)}{{ }^{\frac{1}{\mathrm{~b}}}}\right.$ & Chirife e Iglesias (1978) \\
\hline & Oswin & $\mathrm{m}=\mathrm{a}\left[\frac{\mathrm{a}_{\mathrm{w}}}{1-\mathrm{a}_{\mathrm{w}}}\right]$ & Chirife e Iglesias (1978) \\
\hline & Halsey & $\mathrm{m}=\left[\frac{-\mathrm{a}}{\mathrm{ln} a}\right]^{\frac{-}{\mathrm{b}}}$ & Chirife e Iglesias (1978) \\
\hline & Smith & $\mathrm{m}=\mathrm{a}-\mathrm{b} \cdot \ln \left(1-\mathrm{a}_{\mathrm{w}}\right)$ & Chirife e Iglesias (1978) \\
\hline & Mizrahi & $a_{w}=\frac{a+m}{b+m}$ & Chirife e Iglesias (1978) \\
\hline \multirow{6}{*}{ 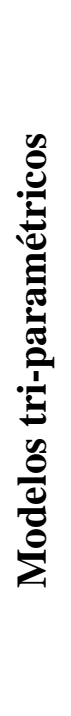 } & GAB & $\mathrm{m}=\frac{\mathrm{m}_{\mathrm{o}} \cdot \mathrm{c} \cdot \mathrm{k} \cdot \mathrm{a}_{\mathrm{w}}}{\left[\left(1-\mathrm{k} \cdot \mathrm{a}_{\mathrm{w}}\right) \cdot\left(1+(\mathrm{c}-1) \cdot \mathrm{k} \cdot \mathrm{a}_{\mathrm{w}}\right)\right]}$ & Maroulis et al. (1988) \\
\hline & BET modificada & $\mathrm{m}=\frac{\mathrm{m}_{\mathrm{o}} \cdot \mathrm{c} \cdot \mathrm{a}_{\mathrm{w}}}{\left[\left(1-\mathrm{k} \cdot \mathrm{a}_{\mathrm{w}}\right) \cdot\left(1+(\mathrm{c}-\mathrm{k}) \cdot \mathrm{a}_{\mathrm{w}}\right)\right]}$ & Jaafar e Michalowski (1990) \\
\hline & Hailwood e Horrobin & $\mathrm{m}=\frac{\mathrm{a}_{\mathrm{w}}}{\left(\mathrm{a}+\mathrm{b} \cdot \mathrm{a}_{\mathrm{w}}+\mathrm{c} \cdot \mathrm{a}_{\mathrm{w}}^{2}\right)}$ & Chirife e Iglesias (1978) \\
\hline & Anderson & $\mathrm{m}=\frac{\mathrm{m}_{\mathrm{o}} \cdot \mathrm{c} \cdot \mathrm{k} \cdot \mathrm{a}_{\mathrm{w}}}{\left[1+(\mathrm{c}-2) \cdot \mathrm{k} \cdot \mathrm{a}_{\mathrm{w}}+(1-\mathrm{c}) \cdot \mathrm{k}^{2} \cdot \mathrm{a}_{\mathrm{w}}^{2}\right]}$ & Boquet et al. (1980) \\
\hline & Anderson e Hall & $\mathrm{m}=\frac{\mathrm{m}_{\mathrm{o}} \cdot \mathrm{c} \cdot \mathrm{a}_{\mathrm{w}}}{\left[1+(\mathrm{c}-2 \cdot \mathrm{k}) \cdot \mathrm{a}_{\mathrm{w}}+\left(\mathrm{k}^{2}-\mathrm{c} \cdot \mathrm{k}\right) \cdot \mathrm{a}_{\mathrm{w}}^{2}\right]}$ & Boquet et al. (1980) \\
\hline & Gascoyne e Pethig & $\mathrm{m}=\frac{\mathrm{m}_{\mathrm{o}} \cdot \mathrm{c} \cdot \mathrm{k} \cdot \mathrm{a}_{\mathrm{w}}}{\left[1+(\mathrm{c}-2 \cdot \mathrm{k}) \cdot \mathrm{a}_{\mathrm{w}}+(\mathrm{k}-\mathrm{c}) \cdot \mathrm{k}^{2} \cdot \mathrm{a}_{\mathrm{w}}^{2}\right.}$ & Boquet et al. (1980) \\
\hline
\end{tabular}

\section{Resultados e discussões}

\subsection{Isotermas de sorção de umidade}

A fibra residual do abacaxi estudada apresentou isotermas de adsorção e dessorção do Tipo III (Figuras 1 e 2), de acordo com a definição clássica de Salwin (1959). Este comportamento é 
respaldado pela composição do produto, que de acordo com Waughon (2006) apresenta teor de açúcares totais superior a $60 \%$.

Nas Figuras 1 e 2 observa-se que nas duas condições de temperatura o produto apresentou suave diferença entre as isotermas de adsorção e dessorção, na faixa de 0,3 a 0,7 de $a_{w}$, o que pode ser caracterizado como efeito de histerese.

Nas Figuras 3 e 4 são apresentadas as isotermas de adsorção e dessorção, nas duas condições de temperatura estudadas.

Figura 1 - Isotermas de sorção da fibra residual do abacaxi a $25^{\circ} \mathrm{C}$

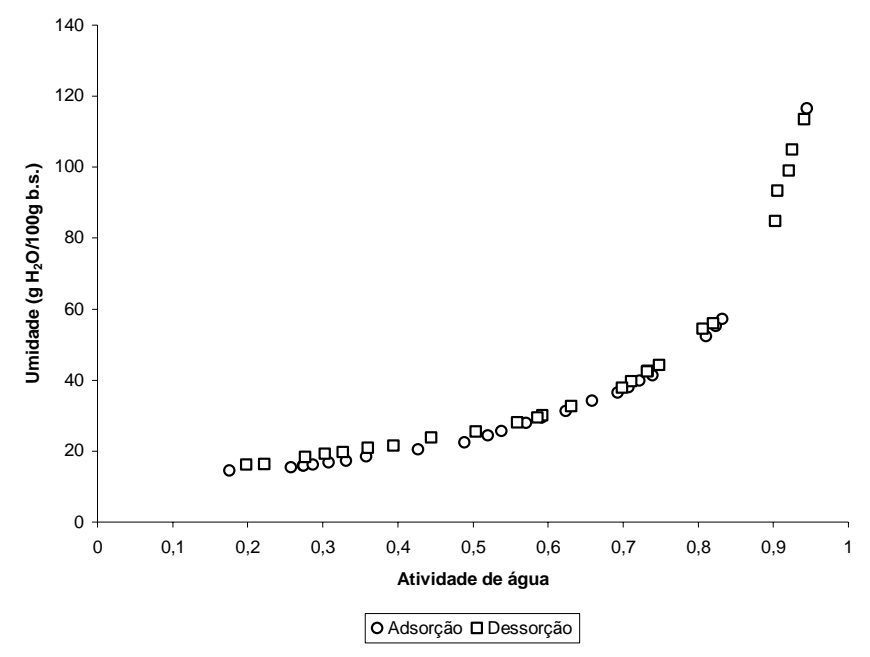

Figura 2 - Isotermas de sorção da fibra residual do abacaxi a $40^{\circ} \mathrm{C}$

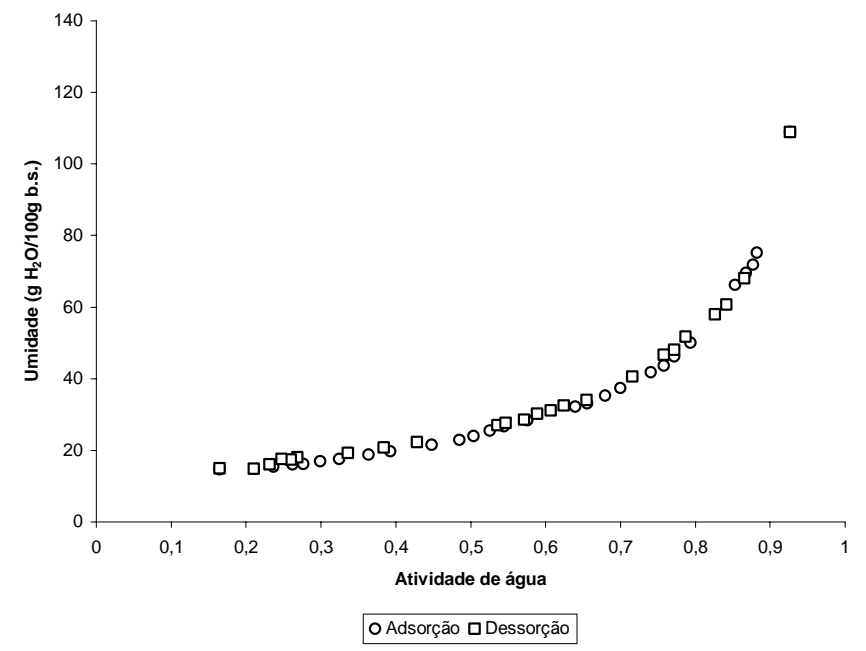


Figura 3 - Isotermas de adsorção da fibra residual do abacaxi

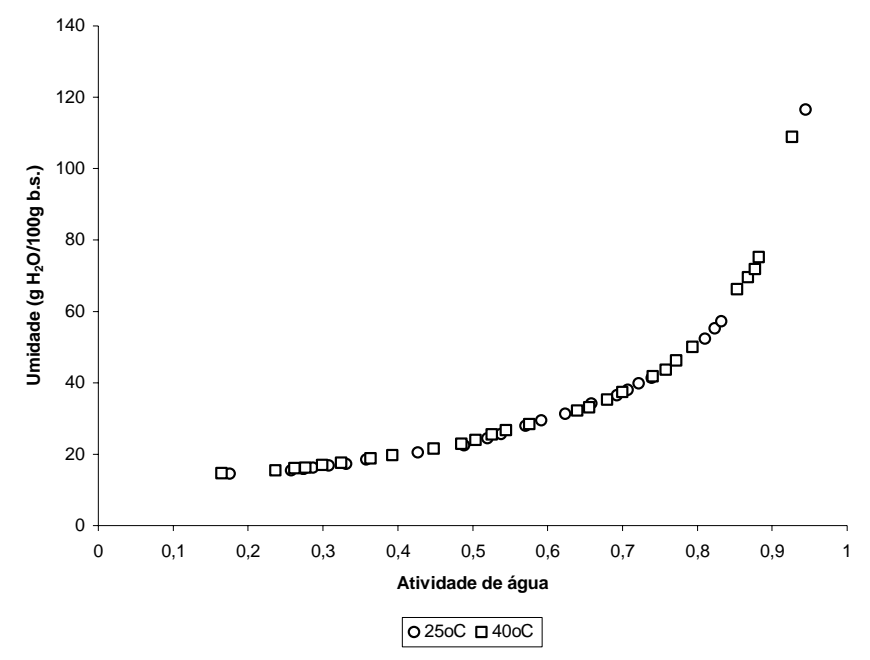

Figura 4 - Isotermas de dessorção da fibra residual do abacaxi

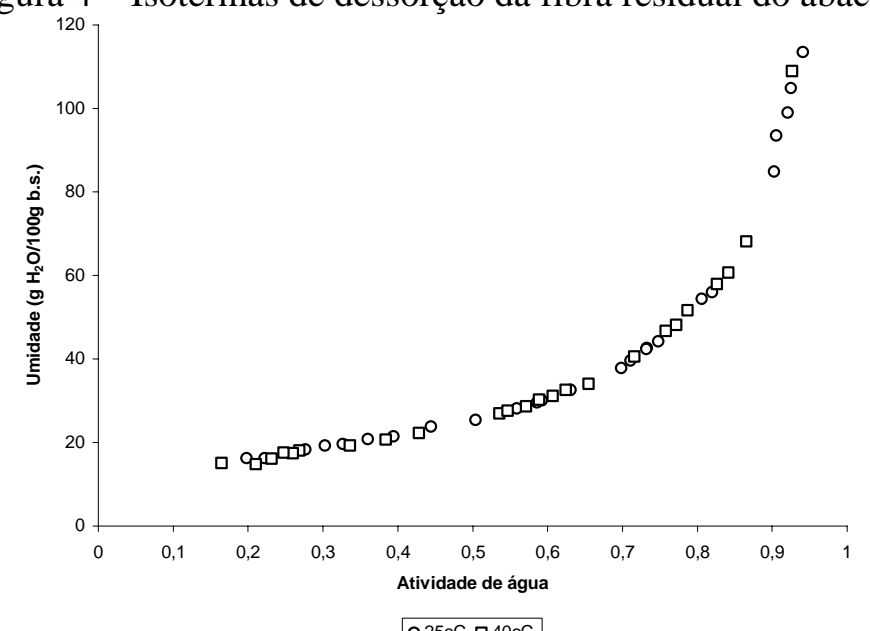

O $250 \mathrm{C} \square 400 \mathrm{C}$

A sobreposição das isotermas obtidas nas diferentes temperaturas, tanto na adsorção quanto na dessorção, indica que não houve efeito da temperatura sobre as mesmas, na faixa estudada. A não existência de efeito significativo foi comprovada estatisticamente, através do cálculo do coeficiente de variação, o qual foi inferior a 5\%. O referido comportamento inviabilizou o cálculo dos calores de dessorção para o produto.

Com base nas isotermas de adsorção (Figura 3) o resíduo seco apresentará estabilidade microbiológica, de acordo com Scott (1957), quando estiver com umidade inferior a 30 g $\mathrm{H}_{2} \mathrm{O} / 100$ g b.s. $\left(\mathrm{a}_{\mathrm{w}}<0,6\right)$. O comportamento das isotermas indica que o produto demandará maiores cuidados, quando armazenado em ambientes com umidades relativas superiores a 70\%; pois acima desse nível o mesmo adsorverá água com muita facilidade. Recomenda-se neste caso a utilização de uma embalagem apropriada (impermeável ao vapor de água).

Durante a obtenção dos dados de sorção não foram observados alterações visualmente perceptíveis no produto, tais como: mudança de cor e aglomeração (perda de fluidez), em toda a faixa de umidade relativa estudada. 


\subsection{Determinação da monocamada}

Os valores médios da monocamada $\left(\mathrm{m}_{\mathrm{o}}\right)$ para adsorção e dessorção foram de 11,6 g $\mathrm{H}_{2} \mathrm{O} / 100$ g b.s. e 12,7 g $\mathrm{H}_{2} \mathrm{O} / 100$ g b.s., respectivamente. Tomando como base o processo de dessorção não se recomenda a secagem do produto a níveis inferiores a 12 g $\mathrm{H}_{2} \mathrm{O} / 100$ g b.s., para evitar gasto desnecessário de energia.

\subsection{Modelagem matemática}

Na Tabela 2 encontram-se os valores dos coeficientes de determinação $\left(\mathrm{r}^{2}\right)$, referentes aos ajustes dos doze modelos matemáticos testados, aos dados de adsorção e dessorção de umidade da fibra residual do abacaxi.

Tabela 2 - Coeficientes de determinação $\left(\mathrm{r}^{2}\right)$ obtidos através dos ajustes

\begin{tabular}{|c|c|c|c|c|c|c|}
\hline & \multirow{2}{*}{ Nome da Equação } & \multicolumn{2}{|c|}{ Adsorção } & \multicolumn{2}{|c|}{ Dessorção } & \multirow{2}{*}{$\mathbf{r}^{2}$ (Médio) } \\
\hline & & $25^{\circ} \mathrm{C}$ & $40^{\circ} \mathrm{C}$ & $25^{\circ} \mathrm{C}$ & $40^{\circ} \mathrm{C}$ & \\
\hline \multirow{6}{*}{ 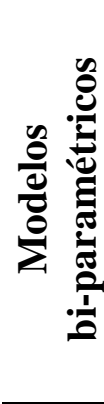 } & Kuhn & 0,9542 & 0,9879 & 0,9766 & 0,9807 & 0,9749 \\
\hline & Handerson & 0,9446 & 0,9514 & 0,9629 & 0,9438 & 0,9507 \\
\hline & Oswin & 0,9957 & 0,9910 & 0,9927 & 0,9868 & 0,9916 \\
\hline & Halsey & 0,9989 & 0,9987 & 0,9961 & 0,9980 & 0,9979 \\
\hline & Smith & 0,9446 & 0,9514 & 0,9629 & 0,9438 & 0,9507 \\
\hline & Mizrahi & 0,9942 & 0,9947 & 0,9906 & 0,9879 & 0,9919 \\
\hline \multirow{6}{*}{ 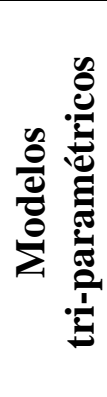 } & GAB & 0,9990 & 0,9974 & 0,9977 & 0,9961 & 0,9976 \\
\hline & BET modificada & 0,9990 & 0,9973 & 0,9977 & 0,9953 & 0,9973 \\
\hline & Hailwood-Horrobin & 0,9990 & 0,9979 & 0,9979 & 0,9964 & 0,9978 \\
\hline & Anderson & 0,9990 & 0,9974 & 0,9974 & 0,9954 & 0,9973 \\
\hline & Anderson-Hall & 0,9990 & 0,9973 & 0,9977 & 0,9957 & 0,9974 \\
\hline & Gascoyne-Pethig & 0,8056 & 0,8397 & 0,8993 & 0,7784 & 0,8308 \\
\hline
\end{tabular}

Analisando apenas os valores de $\mathrm{r}^{2}$ constata-se que os modelos bi-paramétricos de Oswin, Halsey e Mizrahi, e os tri-paramétricos de GAB, BET modificada, Hailwood-Horrobin, Anderson e Anderson-Hall foram os que apresentaram os melhores ajustes; sendo em um primeiro momento os mais indicados na predição das isotermas de sorção do resíduo estudado.

A partir dos desvios médios relativos (P), apresentados na Tabela 3, constata-se que o modelo bi-paramétrico de Halsey ( $\mathrm{P}=2,4 \%$ ) e o tri-paramétrico de Hailwood-Horrobin ( $\mathrm{P}=2,2 \%$ ), são os mais recomendados na predição das isotermas de adsorção e dessorção do produto estudado. 
O modelo de Halsey, por ter apenas dois parâmetros, apresenta mais fácil solução matemática; não necessitando de regressão não linear; sendo nesse caso o mais indicado para utilização. Os valores dos parâmetros dos modelos de Halsey e Hailwood-Horrobin, para as diferentes condições são apresentados na Tabela 4.

São considerados bons ajustes aqueles para os quais o valor de P é inferior a 5\%. Ressaltase ainda que entre os modelos da Tabela 3 o único que apresentou valor de P superior a 5\% foi Oswin, sendo o menos indicado para utilização na predição das isotermas do produto.

Tabela 3 - Desvios médios relativos (P) para os modelos com maiores $\mathrm{r}^{2}$

\begin{tabular}{lccccc}
\hline \multirow{2}{*}{ Nome da Equação } & \multicolumn{2}{c}{ Adsorção } & \multicolumn{2}{c}{ Dessorção } & \multirow{2}{*}{$\mathbf{P}_{\text {(Médio) }}$} \\
\cline { 2 - 5 } & $\mathbf{2 5}^{\circ} \mathbf{C}$ & $\mathbf{4 0}^{\circ} \mathbf{C}$ & $\mathbf{2 5}^{\circ} \mathbf{C}$ & $\mathbf{4 0}^{\circ} \mathbf{C}$ & \\
\hline Oswin & 5,2 & 6,7 & 7,3 & 8,1 & $\mathbf{6 , 8}$ \\
Halsey & 2,6 & 2,1 & 2,5 & 2,4 & $\mathbf{2 , 4}$ \\
Mizrahi & 2,7 & 2,7 & 4,3 & 5,5 & $\mathbf{3 , 8}$ \\
GAB & 2,0 & 2,3 & 2,5 & 2,9 & $\mathbf{2 , 4}$ \\
BET modificada & 2,0 & 2,4 & 2,4 & 3,8 & $\mathbf{2 , 7}$ \\
Hailwood-Horrobin & 2,0 & 2,1 & 1,8 & 3,0 & $\mathbf{2 , 2}$ \\
Anderson & 2,0 & 2,3 & 3,0 & 3,4 & $\mathbf{2 , 7}$ \\
Anderson-Hall & 2,0 & 2,5 & 2,2 & 3,2 & $\mathbf{2 , 5}$ \\
\hline
\end{tabular}

Tabela 4 - Valores das constantes dos modelos de Halsey e Hailwood-Horrobin

\begin{tabular}{lccccc}
\hline \multirow{2}{*}{ Nome da Equação } & \multirow{2}{*}{ Processo } & Temperatura & \multicolumn{3}{c}{ Constantes } \\
\cline { 4 - 6 } & & & $\mathbf{A}$ & $\mathbf{b}$ & $\mathbf{c}$ \\
\hline \multirow{2}{*}{ Halsey } & \multirow{2}{*}{ Adsorção } & $25^{\circ} \mathrm{C}$ & 111,40 & 1,59 & - \\
& & $40^{\circ} \mathrm{C}$ & 75,53 & 1,48 & - \\
& \multirow{2}{*}{ Dessorção } & $25^{\circ} \mathrm{C}$ & 126,07 & 1,60 & - \\
& & $40^{\circ} \mathrm{C}$ & 99,99 & 1,54 & - \\
\hline \multirow{3}{*}{ Hailwood-Horrobin } & \multirow{2}{*}{ Adsorção } & $25^{\circ} \mathrm{C}$ & 0,00133 & 0,0768 & $-0,0737$ \\
& & $40^{\circ} \mathrm{C}$ & $-0,00067$ & 0,0844 & $-0,0801$ \\
& \multirow{2}{*}{ Dessorção } & $25^{\circ} \mathrm{C}$ & $-0,00067$ & 0,0775 & $-0,0725$ \\
& & $40^{\circ} \mathrm{C}$ & $-0,00057$ & 0,0797 & $-0,0752$ \\
\hline
\end{tabular}

\section{Conclusão}

As isotermas de adsorção e dessorção da fibra residual do abacaxi, após secagem, apresentaram um comportamento típico de isotermas do Tipo III.

As isotermas do produto apresentaram suave efeito de histerese na faixa de atividade de água de 0,3 a 0,7 . 
O produto estudado será considerado microbiologicamente estável se apresentar um teor de umidade inferior a $30 \mathrm{~g} \mathrm{H}_{2} \mathrm{O} / 100 \mathrm{~g}$ b.s.

O produto demandará maiores cuidados quando armazenado em ambientes com umidades relativas superiores a 70\%, pois adsorverá água com maior facilidade.

Os modelos bi-paramétricos de Oswin, Halsey e Mizrahi, e os tri-paramétricos de GAB, BET modificada, Hailwood-Horrobin, Anderson e Anderson-Hall podem ser utilizados na predição das isotermas de sorção de umidade da fibra residual do abacaxi, nas condições estudadas e com margens de erro aceitáveis.

Entre todos os modelos testados o bi-paramétrico de Halsey e o tri-paramétrico de Hailwood-Horrobin são preferencialmente os mais indicados para serem utilizados na predição das isotermas de adsorção e dessorção da fibra residual do abacaxi.

\begin{abstract}
It was evaluated the hygroscopic behavior of pineapple residual fiber, dried in fix-bed dryer. It was plotted adsorption and desorption isotherm at $25^{\circ} \mathrm{C}$ and $45^{\circ} \mathrm{C}$ and determined BET monolayer value $\left(\mathrm{m}_{\mathrm{o}}\right)$. There were fitted twelve mathematics models to adsorption and desorption experimental data. The product presented the sorption isotherm Type III. The values of monolayer obtained to adsorption and desorption were $11.6 \mathrm{~g} \mathrm{H}_{2} \mathrm{O} / 100 \mathrm{~g}$ dry base and $12.7 \mathrm{~g} \mathrm{H}_{2} \mathrm{O} / 100 \mathrm{~g}$ dry base, respectively. The best mathematic models fitted to prediction of adsorption and desorption isotherm, in the analyzed conditions, were: Oswin, Halsey and Mizrahi models (bi-parameters); GAB, BET modified, Hailwood-Horrobin, Anderson and Anderson-Hall models (tri-parameters).
\end{abstract}

Keywords: isotherms; sorption; residue; pineapple.

\title{
Referências
}

BOQUET, R.; CHIRIFE, J.; IGLESIAS, H.A. On the equivalence of isotherm equations. Journal of Food Technology, v.15, p.345-349, 1980.

BRUNAUER, S.; EMMET, P.H.; TELLER, E. Adsorption of gases in multimolecular layers. Journal American Chemical Society, v.60, p.309-319, 1938.

CHIRIFE, J.; IGLESIAS, H.A. Equations for fitting water sorption isotherms of foods: Part 1 - A review. Journal of Food Technology, v.13, p.159-174, 1978.

JAAFAR, F.; MICHALOWSKI, S. Modified BET equation for sorption/desorption isotherms. Drying Technology, v.8, n.4, p.811-127, 1990.

LABUZA, T.P. Sorption phenomena in foods. Food technology, v.22, n.263, p.15-24, 1968.

LOMAURO, C.J.; BAKSHI, A.S.; LABUZA, T.P. Evaluation of food moisture sorption isotherm equations. Part I: fruit, vegetable and meat products. Lebensmittel Wissenschaft und Technologie, v.18, n.2, p.111-117, 1985a.

LOMAURO, C.J.; BAKSHI, A.S.; LABUZA, T.P. Evaluation of food moisture sorption isotherm equations. Part II: milk, coffee, tea, nuts, oilseeds, spices and starchy foods. Lebensmittel Wisseschaft und Technologie, v.18, n.2, p.118-124, 1985b.

MAROULIS, Z.B.; TSAMI, E.; ARINOS-KOURIS, D.; SARAVACOS, G.D. Application of the GAB model to the sorption isotherms for dried fruits. Journal of Food Engineering, v.7, p.63-70, 1988. 
SALWIN, H. Defining minimum moisture contents for dehydrated foods. Food Technology, v.13, p.594-585, 1959.

SCOTT, W.J. Water relations of food spoilage microorganisms. Advents Food Research, v.7, p.83-127, 1957.

STATISTICA for Windows 5.5. Computer program manual. Tulsa: StatSoft, Inc., 2000.

UBOLDI EIROA, M.N. Atividade de água: Influência sobre o desenvolvimento de microrganismos e métodos de determinação em alimentos. Boletim do ITAL, campinas, p.353-381, 1981.

WAUGHON, T.G.M. Caracterização e processamento do resíduo fibroso gerado na industrialização do suco de abacaxi. 2006. 63f. Dissertação (Mestrado em Ciência e Tecnologia de Alimentos) - Universidade Federal do Pará. Belém, 2006.

\section{AGRADECIMENTOS}

A Financeira de Estudos e Projetos - FINEP, pelo apoio financeiro indispensável à execução deste trabalho e ao Conselho Nacional de Desenvolvimento Científico e Tecnológico CNPq, pela concessão da bolsa de mestrado.

\section{Dados do segundo autor:}

\section{Rosinelson da Silva Pena}

Faculdade de Engenharia de Alimentos/Instituto de Tecnologia/Universidade Federal do Pará Professor

Conj. Médice II - Rua Cafezal, 175 - Marambaia - CEP 66620-190 - Belém - PA

Tel. (91) 3201-7626 Fax (91)3201-7456

rspena@ufpa.br 